Original

Article

\section{Spironolactone: A promising anti-schistosomal drug as revealed by scanning electron microscopy of adult worms}

\author{
Heba A Aminou, Abeer A Abdel Rahman
}

Medical Parasitology Department, Faculty of Medicine, Ain-Shams University, Cairo, Egypt

\begin{abstract}
Background: Schistosomiasis mansoni is still a widespread parasitic infection especially among poor developing countries. Depending on praziquantel (PZQ) as a single medication for treatment and control, causes the emergence of isolates resistant to this drug. Continuous efforts are needed for the introduction of new effective therapies. Some diuretics including spironolactone, showed promising anti-parasitic effects.

Objectives: In this study, spironolactone, a potassium-sparing diuretic, was evaluated regarding its in vitro lethal effect on Schistosoma mansoni (S. mansoni) adult worms using scanning electron microscopy (SEM).

Material and Methods: A total of 144 worms; 72 males and 72 females were exposed to a concentration of $10 \mu \mathrm{g} / \mathrm{ml}$ of spironolactone for 5 days at $37 \pm 0.5^{\circ} \mathrm{C}$ in $5 \% \mathrm{CO}_{2}$ incubator. A pure medium and a medium containing $0.5 \%$ of dimethyl sulfoxide (DMSO) (vehicle) were used as negative controls, while PZQ at $10 \mu \mathrm{g} / \mathrm{ml}$ was used as a reference drug. The compound was then retested using the same technique by successive descending dilutions of the solution. Mortality rate of $50 \%$ (lethal concentration) (LC50) and $100 \%$ (lethal concentration) (LC100) were calculated after $72 \mathrm{~h}$ and 5 days exposure.

Results: Our results highlighted the potent anti-schistosome effect of spironolactone as it showed 100\% mortality of worms at $6.4 \mu \mathrm{g} / \mathrm{ml}$ and $5 \mu \mathrm{g} / \mathrm{ml}$ after $72 \mathrm{~h}$ and 5 days exposure, respectively. While $50 \%$ mortality of worms was obtained at $4.5 \mu \mathrm{g} / \mathrm{ml}$ and $3 \mu \mathrm{g} / \mathrm{ml}$ after $72 \mathrm{~h}$ and 5 days exposure, respectively. Moreover, SEM of male and female $S$. mansoni worms exposed to spironolactone in vitro, showed disintegration and sloughing of the tubercles on the dorsal surface of males, erosion of the tegument with exposure and appearance of the sub-tegumental tissue in males and females.

Conclusion: Being a safe, oral, inexpensive, and well tolerated drug, spironolactone is capable of being a potent future anti-schistosome drug.
\end{abstract}

Keywords: praziquantel, scanning electron microscopy, schistosomiasis mansoni, spironolactone.

Received: 18 June, 2020, Accepted: 17 August, 2020.

Corresponding Author: Heba A Aminou, Tel.: +20 1001550924, E-mail: hebaaminou74@hotmail.com

Print ISSN: 1687-7942, Online ISSN: 2090-2646, Vol. 13, No. 2, August, 2020.

\section{INTRODUCTION}

Schistosomiasis is still a serious public health problem that affects 230 million people worldwide, especially in tropical and subtropical areas with poor sanitary conditions ${ }^{[1]}$. It is one of the most significant, neglected tropical diseases in the world due to infection by intravascular trematode flatworms of the genus Schistosoma ${ }^{[2]}$. About 80 million infections by $S$. mansoni were reported in Africa, the Near East, and South America causing intestinal schistosomiasis ${ }^{[3]}$. Presence of parasite eggs in the tissues is the main issue of the disease causing chronic inflammatory response to Schistosoma ova with granuloma formation leading to periportal fibrosis and portal hypertension manifestations ${ }^{[1]}$.

Praziquantel (PZQ) is the only recommended drug for schistosomiasis, but still, it has some disadvantages; the tablets are large, have an unpalatable taste, a large dose is needed ${ }^{[4]}$, and the juvenile forms of the parasite are not affected ${ }^{[5]}$. Increasing PZQ resistance against some isolates of $S$. mansoni has been recorded as a result of widespread use of the drug ${ }^{[6]}$. Thus, the scientific community was concerned with the development of new, cheap and safe anti-schistosomiasis drugs ${ }^{[7]}$.

Diuretics are the most commonly indicated drugs for treatment of fluid retention, especially in cases of heartfailure, renal failure and liver cirrhosis ${ }^{[8]}$. They are generally well tolerated, cheap, orally administered, and safe $^{[9]}$. Spironolactone, a potassium-sparing diuretic, is a mineralocorticoid receptor antagonist, specifically an antagonist of aldosterone ${ }^{[10]}$; and is a very effective agent in the treatment of heart failure $^{[11]}$, myocardial infarction ${ }^{[12]}$ and resistant hypertension ${ }^{[13]}$. Moreover, together with short-term course of corticosteroids, spironolactone was used in the treatment of resistant ascites complicating schistosome liver disease ${ }^{[14]}$. In a case of myocarditis due to malarial infection, spironolactone was also 
effective in treating respiratory distress ${ }^{[15]}$. Recently, Guerra et al. ${ }^{[16]}$ showed that spironolactone is able to alter adult $S$. mansoni worm morphology and motor activity, leading to parasitic death. Moreover, it was found that oral treatment with spironolactone in mice harboring infections significantly reduced worm burden, egg production and hepatosplenomegaly ${ }^{[16]}$. In this study, spironolactone was evaluated as regarding its in vitro lethal effect on $S$. mansoni adult worms, and hence, its use as a potential anti-schistosomal drug.

\section{SUBJECTS AND METHODS}

This experimental study was conducted in Theodor Bilharz Research Institute (TBRI) during the period from May-June 2019.

Adult worms: The $S$. mansoni worms as well as the materials used were provided by the Schistosome Biological Material Supply Centre of Theodore Bilharz Research Institute (TBRI), Giza, Egypt. Adult worms were obtained by the main procedure previously described by Yousif et al. ${ }^{[17]}$ and Ramirez et al. ${ }^{[18]}$ from hamsters (Mesocricetus auratus), percutaneously infected with 350-400 S. mansoni cercariae per hamster 6 weeks earlier. The worms were perfused by citrated saline, and the recovered worms were washed from blood in small sieves ( $20 \mu \mathrm{m}$ mesh) by phosphate buffer. Worms were washed three times with the culture medium used for the assay under a sterilized laminar flow chamber. The culture medium was composed of Roswell Park Memorial Institute Medium (RPMI) $1640+$ l-glutamine $+20 \%$ fetal calf serum + antibiotics $(300 \mu \mathrm{g}$ streptomycin +300 IU penicillin + $160 \mu$ g gentamycin per $\mathrm{ml})^{[19]}$.

Bioactivity testing: The bioassay was carried out according to Eissa et al. ${ }^{[19]}$ using 24 wells tissue culture plates. A stock solution $5 \mathrm{mg} / \mathrm{ml}$ of the compound was prepared in DMSO immediately before use. Six worms, three males and three females were introduced into each test well in $2 \mathrm{ml}$ medium. Worms were exposed to a concentration of $10 \mu \mathrm{g} / \mathrm{ml}$ of spironolactone for 5 days at $37 \pm 0.5^{\circ} \mathrm{C}$ in $5 \% \mathrm{CO}_{2}$ incubator (2 replicates were prepared to test spironolactone). A pure medium and a medium containing $0.5 \%$ of DMSO (vehicle) were used as negative controls, while PZQ at $10 \mu \mathrm{g} / \mathrm{ml}$ was used as a reference drug. Worms were examined for their viability using a stereomicroscope, and those not showing motility for one minute were considered dead. The mortality rate of worms was calculated after $72 \mathrm{~h}$ and 5 days exposure. The compound was then retested using the same technique by successive descending dilutions of the solution.

SEM study: Adult worms of $S$. mansoni exposed to spironolactone and PZQ, and non-exposed samples were fixed in a $10 \%$ glutaraldehyde, then processed for examination by $\operatorname{SEM}^{[20]}$.
Statistical analysis: The statistical program SPSS version 20 was used for the calculation. Linear regression analysis was applied to determine the relationship between worm mortality and drug concentration. Probit regression graphing was used to determine LC50 and LC100.

Ethical approval: The study was approved by the Research Ethics Committee, Faculty of Medicine, Ain Shams University, Cairo, Egypt, FWA 00006444, and hamsters were handled according to the NIH guidelines for animal experimentation. The animal experiments were performed according to the national regulations for the Animal Ethics rules, Ain-Shams University, Cairo, Egypt. Hamsters were maintained under conventional conditions, fed a standard commercial pellet diet, housed in $12 \mathrm{~h}$ dark cycle at $22 \pm 2{ }^{\circ} \mathrm{C}$, humidity of $55 \% \pm$ $10 \%$ and continuous air renovation, at the Schistosome Biological Material Supply Centre of Theodore Bilharz Research Institute (TBRI), Giza, Egypt.

\section{RESULTS}

In vitro schistosomicidal activity of spironolactone showed $100 \%$ mortality of worms at $6.4 \mu \mathrm{g} / \mathrm{ml}$ and 5 $\mu \mathrm{g} / \mathrm{ml}$ after $72 \mathrm{~h}$ and 5 days exposure, respectively. While $50 \%$ mortality of worms was obtained at 4.5 $\mu \mathrm{g} / \mathrm{ml}$ and $3 \mu \mathrm{g} / \mathrm{ml}$ after $72 \mathrm{~h}(3$ males and 3 females were dead) and 5 days ( 2 males and 4 females were dead) exposure, respectively. However, this estimation of mortality proved to be lower than that of the reference drug (PZQ) which showed 100\% mortality of worms at $0.4 \mu \mathrm{g} / \mathrm{ml}$ and $0.3 \mu \mathrm{g} / \mathrm{ml}$ after $72 \mathrm{~h}$ and 5 days exposure, respectively. While $50 \%$ mortality of worms was obtained at $0.3 \mu \mathrm{g} / \mathrm{ml}$ and 0.2 $\mu \mathrm{g} / \mathrm{ml}$ after $72 \mathrm{~h}$ and 5 days exposure, respectively, under the same condition (Table 1).

Table 1. In vitro schistosomicidal activity of PZQ and spironolactone on Schistosoma mansoni adult worms (after $72 \mathrm{~h}$ and 5 days exposure).

\begin{tabular}{lcccc}
\hline \hline & \multicolumn{2}{c}{ Praziquantel } & \multicolumn{2}{c}{ Spironolactone } \\
\cline { 2 - 5 } & $\begin{array}{c}\mathbf{L C 5 0} \\
\boldsymbol{\mu g} / \mathbf{m l}\end{array}$ & $\begin{array}{c}\mathbf{L C 1 0 0} \\
\boldsymbol{\mu g} / \mathbf{m l}\end{array}$ & $\begin{array}{c}\mathbf{L C 5 0} \\
\boldsymbol{\mu g} / \mathbf{m l}\end{array}$ & $\begin{array}{c}\mathbf{L C 1 0 0} \\
\boldsymbol{\mu g} / \mathbf{m l}\end{array}$ \\
\hline 72 h & 0.3 & 0.4 & 4.5 & 6.4 \\
5 days & 0.2 & 0.3 & 3.0 & 5.0 \\
\hline \hline
\end{tabular}

In the present work, SEM of adult male S. mansoni worms exposed to spironolactone in vitro, showed disintegration and sloughing of the tubercles on the dorsal surface; erosion of the tegument with exposure and appearance of the sub-tegumental tissue occurred in males and females (Figure 1). These findings were similar to those obtained when worms were exposed to PZQ (Figure 2). While SEM of $S$. mansoni worms from both DMSO and pure medium controls (negative controls) showed normal dorsal tubercles with intact tegument (Figure 3). 

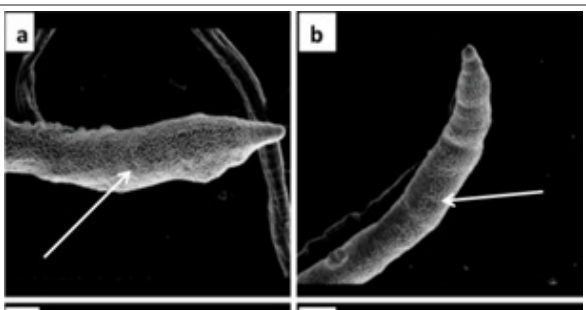

c
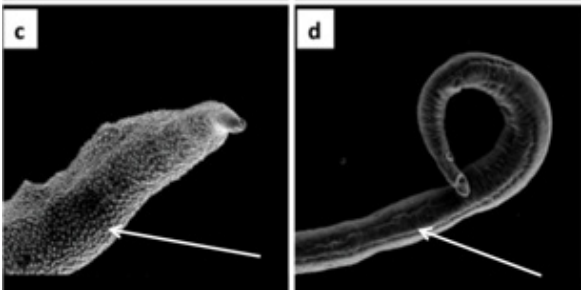

Fig. 1. Scanning electron microscopy (SEM) of adult S. mansoni: in vitro exposed to spironolactone showing disintegration and sloughing of the tubercles on the dorsal surface, erosion of the tegument with exposure and appearance of the subtegumental tissue of both male; (a) (x218), (b) (x218), (c) (x300) and female adult worms; (d) (x380).
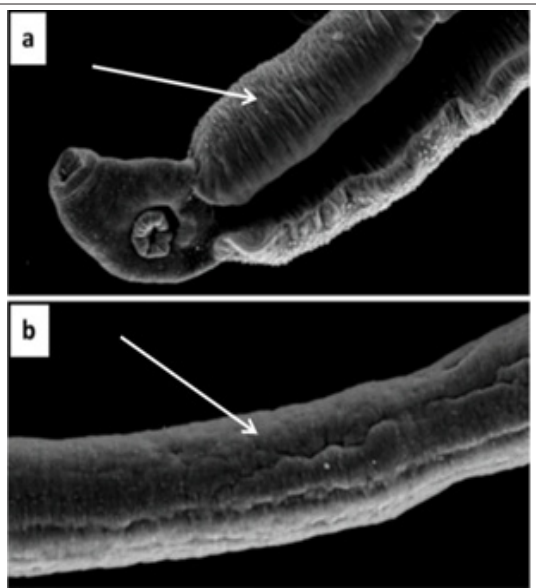

Fig. 2. Scanning electron microscopy (SEM) of adult S. mansoni: in vitro exposed to $\mathrm{PZQ}$ showing complete sloughing of the tubercles on the dorsal surface, erosion of the tegument and exposure of the subtegumental tissue of both male; (a) (x349) and female adult worms; (b) (x600).
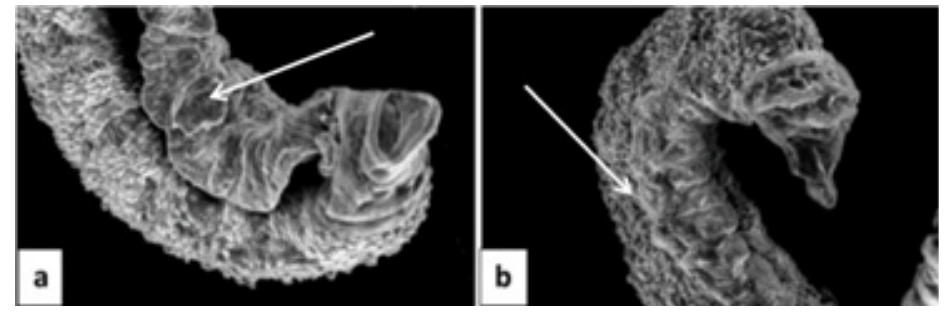

Fig. 3. Unexposed adult male S. mansoni worm showing normal tegument with intact dorsal tubercles at both anterior (a) and posterior ends (b) $(\times 440)$.

\section{DISCUSSION}

Schistosomiasis is one of the most prevalent human parasitic infestations that records high rates of morbidity. Approximately 20 million persons are affected with mortality rate of about 280000 deaths per year, especially in tropical and subtropical regions ${ }^{[21]}$. PZQ is the only commercially available anti-schistosomal drug for the treatment of schistosomiasis ${ }^{[22]}$. However, it is inadvisable to rely on one drug for any infectious condition, especially a disease with high prevalence as schistosomiasis, because of the appearance of drugresistant/ tolerant parasites ${ }^{[23]}$.

Research is now directed towards repurposing of known drugs and evaluation of new drug combinations, in order to reach novel anthelmintics ${ }^{[24}$. In a study conducted by Kamel and Bayaumy ${ }^{[25]}$, it was found that the antimalarial drug, primaquine, possesses moderate anti-schistosomal activity against juvenile and adult $S$. mansoni worms, demonstrated by high mortality and tegumental changes. In 2010, Manneck et $a l^{[26]}$ evaluated the schistosomicidal activity of mefloquine, where SEM observations revealed extensive tegumental destruction, including blebbing, shrinking and sloughing following in vitro incubation of adult $S$. mansoni with the drug. Concerning diuretics, some drugs have shown an anti-parasitic effect, as furosemide, that was found to control intracellular leishmanial growth, and hence, has a therapeutic effect against murine cutaneous leishmaniasis ${ }^{[27]}$; and spironolactone that showed, in a study carried out by Guerra et al. ${ }^{[16]}$, a lethal effect on adult $S$. mansoni worm by altering its morphology and motor activity.

In our in vitro study, we assessed the ability of spironolactone, a potassium-sparing diuretic, as a potential drug for schistosomiasis in comparison with PZQ. The exact toxic effect of spironolactone is at this time not yet clear, except that it has detrimental effect on the worm tegument, resulting in $100 \%$ mortality of worms at drug concentrations of $6.4 \mu \mathrm{g} / \mathrm{ml}$ and $5 \mu \mathrm{g} /$ $\mathrm{ml}$ after $72 \mathrm{~h}$ and 5 days exposure, respectively. While LC50 of worms was obtained at $4.5 \mu \mathrm{g} / \mathrm{ml}$ and $3 \mu \mathrm{g} /$ $\mathrm{ml}$ concentrations after $72 \mathrm{~h}$ and 5 days exposure, respectively. In accordance Guerra et al. ${ }^{[16]}$ tested LC50 of thirteen diuretics including spironolactone against $S$. mansoni worms, and observed that only spironolactone, displayed activity at $50 \mu \mathrm{M}$, with LC50 value of 7.2 $\mu \mathrm{M}$ determined after $72 \mathrm{~h}$. Also, after a series of three independent experiments, authors revealed that schistosomes incubated with spironolactone showed increased motor activity, including contractions, associated with a decrease in body length together with increase in the mortality in a concentration-dependent manner. 
In our study using SEM, it was noticed that spironolactone causes disintegration and sloughing of the tubercles on the dorsal surface of S. mansoni worm; in addition to erosion of the tegument with exposure and appearance of the sub-tegumental tissue. This agrees with Adler ${ }^{[28]}$ who stated that schistosomes' tegument is an important target for drugs. These findings were also analogous with the report of Guerra et al. ${ }^{[16]}$ who observed alteration in tegumental structure in both male and female $S$. mansoni worms that intensified progressively when the concentrations of spironolactone increased. Generally the mentioned morphological alterations were similarly observed in previous studies with other antihelminthic drugs ${ }^{[29,30]}$, including PZQ which exerts a potent in vitro effect against schistosomes causing extensive tegumental alterations in a concentration-dependent manner ${ }^{[31,32]}$.

In conclusion, our study confirms the in vitro potent lethal effect of spironolactone on $S$. mansoni adult worms, supported by SEM findings on examination of dead worms. Thus, spironolactone can be used not only as a diuretic, but also as a schistosomicidal derivative. This work sets the base for further studies on the effect of spironolactone on other stages of $S$. mansoni, as well as S. haematobium; and in vivo on experimentally infected animals.

Authors contribution: Aminou HA designed the plan of work, performed the practical part, analyzed the data, wrote, and revised the manuscript. Abdel-Rahman AA shared in designing the plan of work, analyzing the data, writing, and revising the manuscript.

Financial support: There has been no financial support for this work that could have influenced its outcome.

Conflicts of interest: We wish to confirm that there are no known conflicts of interest associated with this publication. We confirm that the manuscript has been read and approved by all named authors and that there are no other persons who satisfied the criteria for authorship but are not listed. We further confirm that the order of authors listed in the manuscript has been approved by all of us.

\section{REFERENCES}

1. Colley DG, Bustinduy AL, Secor WE, King CH. Human schistosomiasis. Lancet 2014; 383: 2253-2264.

2. Gryseels B, Polman K, Clerinx J, Kestens L. Human schistosomiasis. Lancet 2006; 368: 1106-1118.

3. Mutapi F, Maizels R, Fenwick A, Woolhouse M. Human schistosomiasis in the post mass drug administration era. Lancet Infect Dis 2017; 17:42-48.

4. Stothard JR, Sousa-Figueiredo JC, Betson M, Bustinduy A, Reinhard-Rupp J. Schistosomiasis in African infants and preschool children: Let them now be treated! Trends Parasitol 2013; 29:197-205.
5. Cioli D, Pica-Mattoccia L, Basso A, Guidi A. Schistosomiasis control: praziquantel forever? Mol Biochem Parasitol 2014;195:23-29.

6. Wang W, Wang L, Liang YS. Susceptibility or resistance of praziquantel in human schistosomiasis: a review. Parasitol Res 2012; 111:1871-1877.

7. Seif El-Din SH, El-Lakkany NM, Mohamed MA, Hamed MM, Sterner O, Botros SS. Potential effect of the medicinal plants Calotropis procera, Ficus elastica and Zingiber officinale against Schistosoma mansoni in mice. Pharm Biol 2014; 52(2): 144-150.

8. Sica DA, Gehr TWB, Frishman WH. Use of diuretics in the treatmentof heart failure in older adults. Heart Fail Clin 2017; 13: 503-512.

9. von Lueder TG, Atar D, Krum H. Diuretic use in heart failure and outcomes. Clin Pharmacol Ther 2013; 94:490-498.

10. Manolis AA, Manolis TA, Melita H, Manolis AS. Spotlight on spironolactone oral suspension for the treatment of heart failure: Focus on patient selection and perspectives. Vasc Health Risk Manag J 2019;15: 571-579.

11. Lainscak M, Pelliccia F, Rosano G, Vitale C, Schiariti $\mathrm{M}$, Greco C et al. Safety profile of mineralocorticoid receptor antagonists: spironolactone and eplerenone. Int J Cardiol 2015; 200: 25-29.

12. Papademetriou V, Toumpourleka M, Imprialos KP, Alataki S, Manafis A, Stavropoulos K. The role of mineralo-corticoid receptor antagonists in heart failure with reduced ejection fraction. Curr Pharm Des 2018; 24(46): 5517-5524.

13. Manolis AA, Manolis TA, Melita H, Manolis AS. Eplerenone versus spironolactone in resistant hypertension: an efficacy and/or cost or just a men's issue? Curr Hypertens Rep 2019; 21(3):22.

14. el-Zayadi A, Mohran Z, Hasseeb N, Nagy N, Dabbous H. Short-term course of corticosteroids in the treatment of resistant ascites complicating schistosomal liver disease. Am J Gastroenterol 1991; 86 (1): 53-56.

15. Khan FY. Imported Plasmodium vivax malaria complicated by reversible myocarditis. J Family Community Med 2019; 26(3): 232-234.

16. Guerra RA, Silva MP, Silva TC, Salvadori MC, Teixeira FS, de Oliveira RN et al. In vitro and in vivo studies of spironolactone as an anti-schistosomal drug capable of clinical repurposing. Antimicrob Agents Chemother 2019; 63(3) e01722-18.

17. Yousif F, Hifnawy MS, Soliman G, Boulos L, Labib $\mathrm{TH}$, Mahmoud $\mathrm{S}$ et al. Large-scale in vitro screening of Egyptian native and cultivate plants for schistosomicidal activity. Pharma Biol 2007; 45:501510.

18. Ramirez B, Bickle Q, Yousif F, Mouries MA, Nwaka S. Schistosoma challenge in compound screening. Expert Opin Drug Discov 2007; 2:1-9.

19. Eissa MM, El Bardicy S, Tadros M. Bioactivity of miltefosine against aquatic stages of Schistosoma mansoni, Schistosoma haematobium and their snail hosts, supported by scanning electron microscopy. Parasit Vectors 2011; 4:73-83. 
20. Shaw MK, Erasmus DA. Schistosoma mansoni: structural damage and tegumental repair after in vivo treatment with praziquantel. Parasitology 1987; 94: 243-254.

21. World Health Organization (WHO). Schistosomiasis: Progress Report 2001-2011 and Strategic Plan 20122020. Geneva: World Health Organization Press 2013.

22. Greenberg RM. Schistosome ABC multidrug transporters: from pharmacology to physiology. Int J Parasitol Drugs Drug Resist 2014; 4: 301-309.

23. Gryseels B. Schistosomiasis. Infect Dis Clin North Am 2012; 26: 383-397.

24. Pakharukova MY, Pakharukov YV, Mordvinov VA. Effects of miconazole/clotrimazole and praziquantel combinations against the liver fluke Opisthorchis felineus in vivo and in vitro. Parasitol Res 2018; 117:2327-2331.

25. Kamel ROA, Bayaumy FEA. Ultrastructural alterations in Schistosoma mansoni juvenile and adult male worms after in vitro incubation with primaquine. Mem Inst Oswaldo Cruz, Rio de Janeiro 2017; 112(4): 247-254.

26. Manneck T, Haggenmuller Y, Keiser J. Morphological effects and tegumental alterations induced by mefloquine on schistosomula and adult flukes of Schistosoma mansoni. Parasitology 2010; 137(1):8598.
27. Arruda-Costa N, Escrivani D, Almeida-amaral EE Meyer-fernandes JR. Anti-parasitic effect of the diuretic and NA+-ATPAse inhibitor furosemide in cutaneous leishmaniasis. Parasitology 2017; 144(10): 1375-1383.

28. Adler CE. Dissecting the schistosome cloak. Elife 2018; $7: e 36813$.

29. Silva AP, Silva MP, Oliveira CG, Monteiro DC, Pinto PL, Mendonça RZ et al. Garcinielliptone FC: antiparasitic activity without cytotoxicity to mammalian cells. Toxicol in vitro 2015; 29:681-687.

30. de Brito MRM, Peláez WJ, Faillace MS, Militão GCG, Almeida JRGS, Argüello GA et al. Cyclohexenefused 1,3-oxazines with selective antibacterial and antiparasitic action and low cytotoxic effects. Toxicol in vitro $2017 ; 44: 273-279$.

31. Pica-Mattoccia L, Cioli D. Sex- and stage-related sensitivity of Schistosoma mansoni to in vivo and in vitro praziquantel treatment. Int J Parasitol 2004; 34:527-533.

32. Pinto-Almeida A, Mendes T, de Oliveira RNCSA, Allegretti SM, Belo S, Tomás et al. Morphological characteristics of Schistosoma mansoni PZQ-resistant and -susceptible strains are different in presence of praziquantel. Front Microbiol 2016; 7:594. 